\title{
Factors Affecting the Response to First-Line Treatments in Patients with Anti-N-Methyl-D-Aspartate Receptor Encephalitis
}

\author{
Xiaoting Zhang ${ }^{\mathrm{a}, \mathrm{b}}$ \\ Chunjuan Wang ${ }^{\mathrm{a}}$ \\ Wenyao Zhu ${ }^{\mathrm{c}}$ \\ Baojie Wang ${ }^{d}$ \\ Huiying Liang ${ }^{\mathrm{a}, \mathrm{b}}$ \\ Shougang $\mathrm{Guo}^{\mathrm{a}}$ \\ aDepartment of Neurology, \\ Shandong Provincial Hospital Affiliated \\ to Shandong University, Jinan, China \\ bSchool of Clinical Medicine, \\ Qilu Medical College, \\ Shandong University, Jinan, China \\ 'Department of Radiation Oncology, \\ Yantai Yuhuangding Hospital, Yantai, \\ China \\ ${ }^{\mathrm{d} D e p a r t m e n t ~ o f ~ N e u r o l o g y, ~}$ \\ ENT Hospital Affiliated \\ to Shandong University, Jinan, China
}

\begin{abstract}
Background and Purpose Anti-N-methyl-D-aspartate receptor (anti-NMDAR) encephalitis is the most common type of autoimmune encephalitis. This study aimed to explore the possible factors affecting the response to first-line treatments in patients with anti-NMDAR encephalitis.

Methods We enrolled 29 patients who were diagnosed as anti-NMDAR encephalitis between January 1, 2015, and June 30, 2018. They were divided into the remission and nonremission groups according to their response to first-line treatments. The demographics, clinical manifestations, main ancillary examinations, follow-up treatments, and prognosis of patients were recorded. The symptoms reported on in this study occurred before treatments or during the course of first-line treatments.
\end{abstract}

Results There were 18 patients (62.07\%) in the remission group and 11 patients $(37.93 \%)$ in the nonremission group. Compared to the remission group, a higher proportion of the patients in the nonremission group exhibited involuntary movements, decreased consciousness, central hypoventilation, lung infection, and hypoalbuminemia. The nonremission group had a high incidence of increased intracranial pressure and significant elevations of the neutrophilto-lymphocyte ratio in peripheral blood (NLR), aspartate aminotransferase, and fibrinogen. Six patients (54.55\%) in the nonremission group received second-line immunotherapy. Only one patient (3.45\%) died, which was due to multiple- organ failure.

Conclusions Anti-NMDAR-encephalitis patients with more symptoms-especially involuntary movements, disturbance of consciousness, central hypoventilation, and accompanying hypoalbuminemia and pulmonary infection-may respond poorly to first-line treatments. Positive second-line immunotherapy therefore needs to be considered. Admission to an intensive-care unit, increased cerebrospinal fluid pressure, and increased NLR might be the significant factors affecting the response to first-line treatments.

Key Words anti-N-methyl-D-aspartate receptor encephalitis, immunotherapy, response.
Received November 5, 2018

Revised March 11, 2019

Accepted March 11, 2019

\section{Correspondence}

Shougang Guo, MD

Department of Neurology,

Shandong Provincial Hospital Affiliated

to Shandong University, No. 324,

Jingwuweiqi Road, Huaiyin District,

Jinan 250021, Shandong Province,

China

Tel +86-0531-68776354

Fax $+86-0531-87937741$

E-mail guoshougang1124@163.com

\section{INTRODUCTION}

Anti-N-methyl-D-aspartate receptor (anti-NMDAR) encephalitis, which was first reported by Dalmau in 2007, ${ }^{1}$ is the most common type of autoimmune encephalitis (AE), with a mortality rate of $8-10 \% .{ }^{1}$ It usually affects children and young women with a clinical spectrum that includes acute or subacute psychiatric symptoms, cognitive disorders, epilepsy, decreased consciousness, and autonomic dysfunction, with or without teratoma. ${ }^{2}$ The confirmed main mechanism is dysfunction of NMDARs due to immunoglobulin G (IgG)-mediated internalization that probably occurs throughout the brain. ${ }^{3}$

(a) This is an Open Access article distributed under the terms of the Creative Commons Attribution Non-Commercial License (https://creativecommons.org/licenses/by-nc/4.0) which permits unrestricted non-commercial use, distribution, and reproduction in any medium, provided the original work is properly cited. 
Anti-NMDAR encephalitis is chiefly mediated by humoral immunity, and so its management focuses on immunotherapy and the detection and removal of tumors. ${ }^{4}$ The first-line treatments include steroids, intravenous immunoglobulin (IVIg), or plasma exchange either alone or in combination. A second-line immunotherapy such as rituximab, cyclophosphamide, or bortezomib can also be applied. ${ }^{5}$

The so-called NEOS score was recently proposed for predicting the 1-year functional status in patients with anti-NMDA receptor encephalitis. ${ }^{6}$ This score includes admission to an intensive-care unit (ICU), treatment delay $>4$ weeks, lack of clinical improvement within 4 weeks, abnormal MRI findings, and a CSF white blood cell (WBC) count $>20$ cells $/ \mu \mathrm{L}$. A systematic review ${ }^{7}$ of the prognosis in $\mathrm{AE}$ found that altered consciousness, ICU admission, and no use of immunotherapy were associated with a poor prognosis in antiNMDAR encephalitis. A Korean study found that a high albumin level is a predictor of a favorable response to immunotherapy in $\mathrm{AE}^{.}{ }^{8}$ Our team has recently reported that the neutrophil-to-lymphocyte ratio in peripheral blood (NLR) is a practical and reliable biomarker for monitoring the disease progression in patients with AE. ${ }^{9}$

All of the above-mentioned studies considered possible predictors of the long-term prognosis in patients with $\mathrm{AE}$, but no previous study has focused on the therapeutic response to first-line treatments in patients with anti-NMDAR encephalitis. A large-scale observational cohort study found that $47 \%$ of patients did not improve following first-line treatments and so needed to receive a second-line immunotherapy that included rituximab or cyclophosphamide alone or in combination. ${ }^{5}$ It is essential to identify patients who respond poorly to first-line treatments so that second-line immunotherapy can be administered in a timely manner.

In this study we summarized the clinical features of patients with anti-NMDAR encephalitis who did not exhibit clinical improvement after receiving first-line treatments, with the aim of identifying the factors influencing the response to first-line treatments.

\section{METHODS}

\section{Patient eligibility and grouping}

This retrospective study enrolled 29 patients who were diagnosed as anti-NMDAR encephalitis in the Department of Neurology of Shandong Provincial Hospital Affiliated to Shandong University between January 1, 2015, and June 30, 2018. The diagnostic criteria were as follows: i) the rapid onset (less than 3 months) of at least one of the following six major groups of symptoms: 1) abnormal (psychiatric) behavior or cognitive dysfunction, 2) speech dysfunction (pressured speech, verbal reduction, or mutism), 3) seizures, 4) movement disorder, dyskinesias, or rigidity/abnormal postures, 5) decreased level of consciousness, 6) autonomic dysfunction, or central hypoventilation; ii) positivity for IgG antiNMDAR antibodies; and iii) reasonable exclusion of other disorders.

Patients included in the remission group had responded to first-line treatments when methylprednisolone was reduced to oral intake $(60 \mathrm{mg})$ and IVIg was finished within one round. We defined the nonremission group as the absence of a sustained improvement or the modified Rankin scale (mRS) score remaining at 4 or higher. ${ }^{5}$

The study was approved by the Research Ethics Committee of Shandong Provincial Hospital Affiliated to Shandong University (IRB No. LCYJ: NO.2017-015).

\section{Data collection}

The clinical data of the 29 enrolled patients that were collected retrospectively included sex, age, clinical symptoms, presence of teratoma or other tumors, cranial MRI findings, EEG, antibody titers in serum and CSF (used to classify the samples as negative, weak positive, positive, and strong positive), pressure in a lumbar puncture, and number of nucleated cells and level of protein in CSF (all of the CSF parameters were obtained before first-line treatments). The symptoms were categorized into six groups based on the above-mentioned diagnostic criteria. ${ }^{10}$ and all occurred before treatment or during the course of first-line treatments. Malignancy investigations included CT of the abdomen and chest as well as ultrasonography of the reproductive organs. Whole-body PET with whole-body CT was performed when necessary.

Other routine blood examinations focused on the following indicators: NLR, liver function test [aspartate aminotransferase (AST), alanine aminotransferase, and glutamyltranspeptidase], uric acid (UA), D-dimer, fibrinogen, creatinine, thyroid function, homocysteine, and cystatin C (CysC).

All of the patients enrolled in this study received the following standard first-line treatments: high-dose methylprednisolone (initial dose of 1,000 $\mathrm{mg}$ with halving every 3 days until it was reduced to $60 \mathrm{mg}$ orally) plus IVIg $(0.4 \mathrm{~g} / \mathrm{kg} /$ day for 5 days). No plasma exchange was performed because the hospital conditions did not allow this. All of the cancer patients received tumor resection. All of the patients were routinely given mycophenolate mofetil (MMF) as a long-term immunotherapy to prevent recurrence when methylprednisolone was reduced to oral intake. The response to first-line treatments was evaluated before applying MMF therapy. The subsequently applied major second-line treatments included rituximab and bortezomib. The severity of the disease and outcome were assessed using the mRS score. After discharge, 
the outcome was evaluated during a clinical visit to the neurologist or in a telephone follow-up.

\section{Statistical analyses}

SPSS software (version 22.0, IBM Corporation, Armonk, NY, USA) was used for all statistical analyses. All continuous variables that conformed to a normal distribution are expressed as mean $\pm S D$ values, while the other continuous variables are expressed as median and range values. Student's $t$ test was used to analyze intergroup data that conformed to a normal distribution and had a heterogeneous variance; otherwise the Mann-Whitney U test was used. Fisher's exact test was used to analyze categorical variables. Pearson's correlation was applied to the levels of serum albumin and mRS scores. Probability values of $p<0.05$ were assumed to indicate statistically significant differences.

\section{RESULTS}

\section{Clinical characteristics}

The demographic and clinical manifestations are summarized in Table 1 . The 29 included patients comprised 11 males (37.93\%) and 18 females (62.07\%). The application of the grouping criteria resulted in 18 patients $(62.07 \%)$ in the re- mission group and 11 patients (37.93\%) in the nonremission group. The ages of the patients ranged between 13 and 54 years (median 34 years), and did not differ significantly between the two groups. Five female patients had ovarian teratoma, and in all cases the ovarian lesion was removed after admission. No other tumors were found. Compared with the remission group, patients in the nonremission group had more symptoms and a higher proportion of them exhibited involuntary movements, decreased consciousness, and central hypoventilation. Regarding accompanying symptoms, we found no differences in prodromal symptoms such as fever and headache between the two groups; however, the proportion of patients with lung infection and hypoalbuminemia was higher in the nonremission group. Five patients had been in an ICU for supportive treatment, all of whom were in the nonremission group.

\section{Ancillary examinations}

The initial CSF, antibody titers, brain MRI, EEG, and laboratory findings are presented in Table 2. Twelve (41.38\%) of the 29 patients exhibited normal brain MRI findings. The abnormal MRI lesions in T2-weighted or FLAIR images were distributed as follows: eight patients with increased signals in the medial temporal lobe or hippocampus, five patients with in-

Table 1. Demographics and clinical manifestations of patients with anti-NMDAR encephalitis in the remission and nonremission groups

\begin{tabular}{|c|c|c|c|c|}
\hline & Total $(n=29)$ & Remission $(n=18)$ & Nonremission $(n=11)$ & $p$ \\
\hline Age, years & $34[13-54]$ & $35[14-53]$ & $34[13-54]$ & 0.829 \\
\hline \multicolumn{5}{|l|}{ Sex } \\
\hline Male & $11(37.93)$ & $6(33.33)$ & $5(45.45)$ & 0.696 \\
\hline Female & $18(62.07)$ & $12(66.67)$ & $6(54.55)$ & \\
\hline Interval to a definitive diagnosis, days & $20[10-90]$ & $27[10-90]$ & $20[10-60]$ & 0.512 \\
\hline \multicolumn{5}{|l|}{ Tumor } \\
\hline Ovarian teratoma & $5(17.24)$ & $3(16.67)$ & $2(18.18)$ & 1.000 \\
\hline None & $24(82.76)$ & 15 (83.33) & 9 (81.82) & \\
\hline \multicolumn{5}{|l|}{ Accompanying symptoms } \\
\hline Fever & $20(68.97)$ & $11(61.11)$ & 9 (81.82) & 1.000 \\
\hline Headache & $18(62.07)$ & $10(55.56)$ & $8(72.73)$ & 0.449 \\
\hline Hypoalbuminemia & 11 (37.93) & $3(16.67)$ & $8(72.73)$ & 0.005 \\
\hline Lung infection & $12(41.38)$ & $3(16.67)$ & 9 (81.82) & 0.001 \\
\hline Number of six major symptoms & $4[1-6]$ & $3[1-5]$ & $5[2-6]$ & $<0.001$ \\
\hline Mental behavior disorder or cognitive impairment & $26(89.66)$ & $16(88.9)$ & $10(90.91)$ & 1.000 \\
\hline Epileptic seizure & $25(86.21)$ & $16(88.89)$ & $9(81.82)$ & 0.622 \\
\hline Involuntary movement & $10(34.83)$ & $3(16.67)$ & $7(63.64)$ & 0.017 \\
\hline Decreased consciousness & $16(55.17)$ & $6(33.33)$ & 10 (90.91) & 0.006 \\
\hline Speech disturbance & $11(37.93)$ & $7(38.89)$ & $4(36.34)$ & 1.000 \\
\hline Autonomic nervous dysfunction or central hypoventilation & $8(27.59)$ & $1(5.56)$ & $7(63.64)$ & 0.001 \\
\hline Status epilepticus & $4(13.79)$ & $1(5.56)$ & $3(27.27)$ & 0.139 \\
\hline Admission to an intensive-care unit & $5(17.24)$ & $0(0)$ & $5(45.45)$ & 0.004 \\
\hline
\end{tabular}

Data are median [range] or $n(\%)$ values.

NMDAR: N-methyl-D-aspartate receptor. 
Table 2. Findings of the ancillary examinations in patients with anti-NMDAR encephalitis in the two groups

\begin{tabular}{|c|c|c|c|c|}
\hline & Total $(n=29)$ & Remission $(n=18)$ & Nonremission $(n=11)$ & $p$ \\
\hline \multicolumn{5}{|l|}{ MRI findings } \\
\hline Normal & $12(41.38)$ & 9 (33.33) & $3(27.27)$ & \\
\hline Temporal lobe and hippocampus & $8(27.59)$ & $5(27.78)$ & $3(27.27)$ & \\
\hline Cortical lesion & $5(17.24)$ & $2(11.11)$ & $3(27.27)$ & \\
\hline Basal ganglion & $3(10.34)$ & $2(11.11)$ & $1(9.10)$ & \\
\hline Others & 1 & 0 & $1(9.10)$ & \\
\hline Abnormal EEG (information from 18 patients) & 16 & 9 & 7 & \\
\hline \multicolumn{5}{|l|}{ CSF findings } \\
\hline Increased intracranial pressure & $9(31.03)$ & $3(16.67)$ & $6(54.55)$ & 0.048 \\
\hline CSF WBC count $\left(\times 10^{6}\right)$ & $22[2-286]$ & $21[2-286]$ & $25[2-180]$ & 0.498 \\
\hline CSF protein, g/L & $0.28[0.12-0.94]$ & $0.30[0.12-0.94]$ & $0.28[0.21-0.59]$ & 0.491 \\
\hline CSF NMDAR antibody titers & & & & 0.643 \\
\hline Negative & 0 & 0 & 0 & \\
\hline Weak positive & $4(13.79)$ & $3(16.67)$ & $1(9.10)$ & \\
\hline Positive & $7(24.14)$ & $5(27.78)$ & $2(18.18)$ & \\
\hline Strong positive & $18(62.07)$ & $10(55.56)$ & $8(72.73)$ & \\
\hline Serum NMDAR antibody titers & & & & 0.247 \\
\hline Negative & $8(27.59)$ & $4(22.22)$ & $4(36.36)$ & \\
\hline Weak positive & $13(44.83)$ & $10(55.56)$ & $3(27.27)$ & \\
\hline Positive & $4(13.79)$ & $3(16.67)$ & $1(9.10)$ & \\
\hline Strong positive & $4(13.79)$ & $1(5.56)$ & $3(27.27)$ & \\
\hline$N L R_{1}$ & $2.66[1.32-9.86]$ & $2.39[1.32-4.43]$ & $4.10[1.64-9.86]$ & 0.011 \\
\hline $\mathrm{NLR}_{2}$ & $3.07[1.71-19.56]$ & $2.62[1.71-3.86]$ & $4.14[1.94-19.56]$ & 0.003 \\
\hline Albumin $_{1}$ & 42.20 [30.50-47.30] & $41.80[35.40-45.30]$ & $42.30[30.50-47.30]$ & 0.431 \\
\hline Albumin $_{2}$ & 36.30 [16.90-43.90] & 38.50 [32.60-43.20] & 32.70 [16.90-43.90] & 0.012 \\
\hline AST & 20.00 [12.00-93.00] & 17.50 [12.00-66.00] & 31.00 [14.00-93.00] & 0.030 \\
\hline ALT & $19.00[8.00-86.00]$ & $16.50[8.00-86.00]$ & 22.00 [10.00-80.00] & 0.368 \\
\hline GGT & $22.00[12.00-95.00)]$ & $18.50[12.00-46.00]$ & $22.00[15.00-95.00]$ & 0.148 \\
\hline Homocysteine & 11.70 [4.60-97.60] & 11.20 [4.6-97.60] & $12.30[4.600-25.30]$ & 0.904 \\
\hline Uric acid & $258 \pm 118$ & $246 \pm 86$ & $276 \pm 157$ & 0.525 \\
\hline CysC & $0.79 \pm 0.24$ & $0.85 \pm 0.25$ & $0.70 \pm 0.19$ & 0.094 \\
\hline Creatinine & $54.26 \pm 12.56$ & $54.91 \pm 13.04$ & $53.37 \pm 12.46$ & 0.763 \\
\hline FT3 & $3.84 \pm 0.88$ & $3.99 \pm 0.71$ & $3.58 \pm 1.09$ & 0.296 \\
\hline FT4 & $16.64 \pm 3.53$ & $16.41 \pm 4.17$ & $17.00 \pm 2.33$ & 0.722 \\
\hline TSH & $1.34 \pm 1.09$ & $1.54 \pm 1.16$ & $1.01 \pm 0.92$ & 0.292 \\
\hline D-dimer & $0.59[0.15-3.66]$ & $0.36[0.15-1.80]$ & $0.69[0.19-3.66]$ & 0.052 \\
\hline Fibrinogen & $3.26 \pm 0.86$ & $2.99 \pm 0.76$ & $3.80 \pm 0.84$ & 0.026 \\
\hline
\end{tabular}

Data are median [range], mean \pm SD, or $n(\%)$ values.

Albumin : albumin level before first-line treatments, Albumin 2 : albumin level after first-line treatments, ALT: alanine aminotransferase, AST: aspartate aminotransferase, CysC: cystatin C, FT3: free tri-iodothyronine, FT4: serum free thyroxin, GGT: glutamyltranspeptidase, NLR: neutrophil-to-lymphocyte ratio in peripheral blood, NLR : NLR before first-line treatments, NLR $:$ NLR after first-line treatments, NMDAR: N-methyl-D-aspartate receptor, TSH: thyroid-stimulating hormone, WBC: white blood cell.

creased signals in the cerebral cortex, three patients with increased signals in the basal ganglia, and one patient with contrast enhancement of the optic nerve. The distribution of intracranial lesions did not differ between the two groups.

The EEG findings were abnormal in 16 (88.89\%) of the 18 patients for whom EEG information was available. Most EEGs were collected during the seizure interval, and almost all of the patients had bilateral or unilateral generalized slow waves, while one patient exhibited diffuse beta activities and no epileptiform discharges were found.

The intracranial pressure was higher than $180 \mathrm{~mm} \mathrm{H}_{2} \mathrm{O}$ in nine patients, most of whom were in the nonremission group $(p=0.048)$. No significant difference was found in leukocyte and protein levels in CSF. Eight patients were negative for NM- 
DAR antibody in serum but none of the 29 patients showed negativity in CSF. The distribution of the antibody titers in all of the patients is presented in Table 2; there were no differences between the two groups.

We calculated the NLR before and after first-line treatments in both groups of patients. The NLR was lower in the remission group than the nonremission group before and after the first-line treatments ( $p=0.011$ and $p=0.003$, respectively) (Fig. 1). The albumin levels after the first-line treatments were lower in the nonremission group than the remission group, and the changes in these levels were associated with changes in the mRS scores (Fig. 2). A lack of data meant that information on liver enzyme indicators, UA, D-dimer, fibrinogen, creatinine, homocysteine, $\mathrm{CysC}$, and thyroid function was only collected before the first-line treatments. For the remaining collected laboratory indicators, only AST and fibrinogen were significantly elevated in the nonremission group.

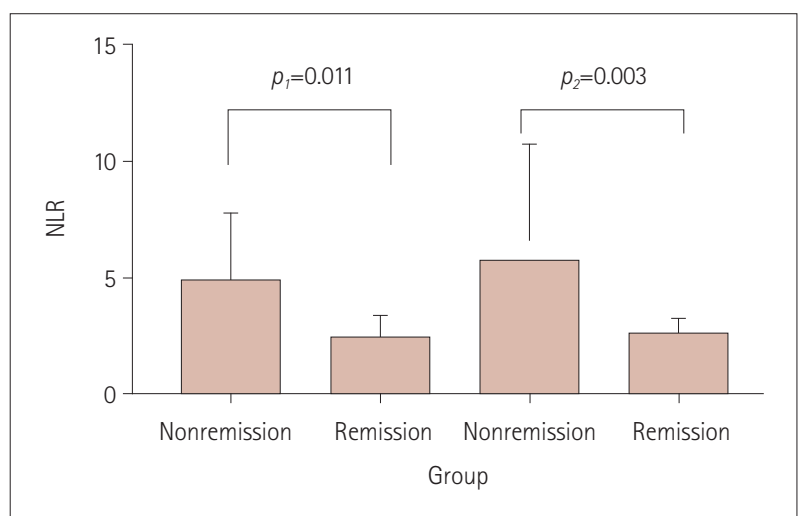

Fig. 1. Differences in the NLR between the remission and nonremission groups. Data are mean and standard-deviation values. NLR: neutrophilto-lymphocyte ratio in peripheral blood, $p_{1}$ : before the first-line treatments, $p_{2}$ : after the first-line treatments.

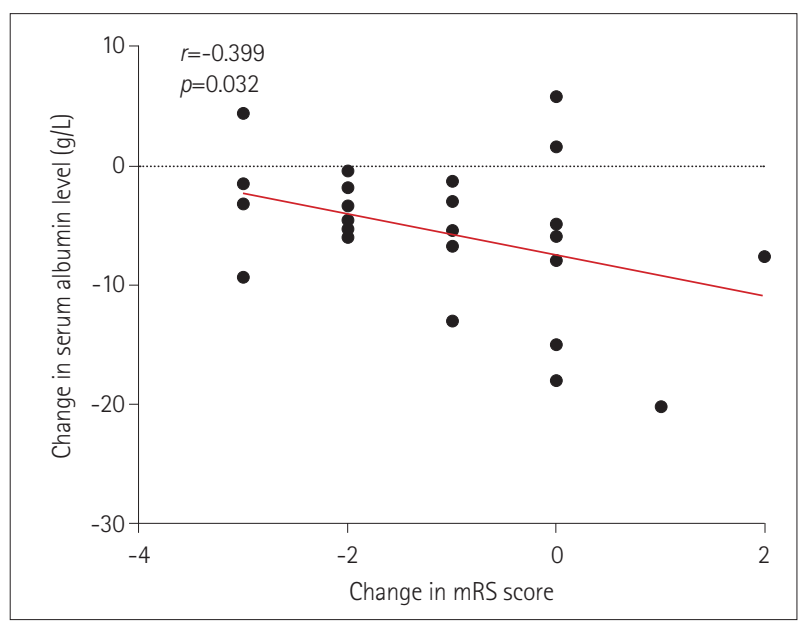

Fig. 2. Correlation between changes in albumin level and $\mathrm{mRS}$ scores. mRS: modified Rankin scale.

\section{Follow-up treatments and prognosis}

Six patients (54.55\%) in the nonremission group received lowdose rituximab, two of whom also received bortezomib. Four patients did not receive second-line immunotherapy due to economic reasons or intolerance. All of them were still in a state of severe neurological deficit at discharge. Only one patient $(3.45 \%)$ died, which was due to multiple- organ failure. At a 1-year follow-up, only two patients did not reach the evaluation standard for a favorable outcome ( $\mathrm{mRS}$ score $<3$ ). Four patients had relapsed at the follow-up, two of whom were in the nonremission group and had not received second-line treatments such as rituximab.

\section{DISCUSSION}

Anti-NMDAR encephalitis is a type of autoimmune disease that can be reversed by early active treatment. This study analyzed the differences between anti-NMDAR-encephalitis patients in remission and nonremission groups after they received first-line treatments, and identified the possible factors affecting the responses to first-line treatments.

According to previous researches, anti-NMDAR encephalitis is mainly found in young females. ${ }^{1}$ In the present study, the median age was 34 years and $62.07 \%$ of the patients were female, with no sex or age difference between the two groups. In the largest observational cohort study so far, $38 \%$ of the anti-NMDAR-encephalitis patients had tumors, $94 \%$ of which were ovarian teratomas. However, in our study only five patients (17.24\%) had ovarian teratomas, which might have been due to the small number of included patients.

We found that the presence of a larger number of symptoms is associated with a lower sensitivity to first-line treatments $(p<0.01)$. Abnormal (psychiatric) behavior and cognitive dysfunction are common symptoms in anti-NMDAR encephalitis. ${ }^{10}$ This was also true in our study, but we did not find differences in the incidence of these features between the two groups. Seizures are another common symptom of antiNMDAR encephalitis. Although the incidence rates of epilepsy and status epilepticus did not differ between the two groups, only one of the four patients with status epilepticus responded to the first-line treatments. We therefore speculate that status epilepticus affects the response to first-line treatments in patients with anti-NMDAR encephalitis. Wang et al. ${ }^{11}$ found that children with anti-NMDAR encephalitis who received both first-line and second-line immunotherapy had a higher incidence of autonomic instability, decreased consciousness, and pediatric ICU stay. An observational study of $111 \mathrm{pa}-$ tients ${ }^{12}$ found that a higher proportion of those in the severe group exhibited involuntary movements, disturbance of consciousness, autonomic dysfunction, and central hypoven- 
tilation. It was recently proposed that ICU admission, treatment delay $>4$ weeks, lack of clinical improvement within 4 weeks, and the presence of movement disorder and central hypoventilation were associated with the 1-year functional status in AE. ${ }^{6}$ Our research findings are basically consistent with those findings, but we also found that the time from onset to diagnosis did not differ between the two groups. A previous study indicated that orofacial-lingual dyskinesia, tremor, and dystonia were the common types of involuntary movements in anti-NMDAR encephalitis. ${ }^{13}$ Histological data have revealed the distribution of NMDAR in the brainstem, but the cause of central hypoventilation in patients with anti-NMDAR encephalitis remains unclear-it may be associated with secondary brainstem lesions or a functional neurotransmitter imbalance. ${ }^{14,15}$ Severe patients exhibiting decreased consciousness and central hypoventilation generally require artificialairway- and ventilator-assisted breathing, while involuntary movements of the face and dystonia may have a considerable impact on airway management. ${ }^{12}$

A Korean study found that a high albumin level is a predictor of a favorable response to immunotherapy in AE. ${ }^{8}$ Our study has confirmed this view from another perspective, by observing that hypoalbuminemia was more likely to occur in the nonremission group, and a significant correlation being found between a decrease in the albumin level and the changes in mRS scores $(r=-0.399, p=0.032)$. A low albumin level is also associated with a poor prognosis in Guillain-Barré syndrome. ${ }^{16}$ The serum albumin levels might be an indicator of IVIg pharmacokinetics, which would result in the serum albumin level indirectly representing the recycling capacity of the neonatal Fc receptor as a target of IVIg. Similarly, a combined lung infection and admission to an ICU are possible indicators of an NMDAR-encephalitis patient not responding to first-line treatments, which are reportedly risk factors for mortality. ${ }^{17}$

Dalmau et al. ${ }^{2,18}$ described that the distribution of intracranial lesions in patients with anti-NMDAR encephalitis was not specific, with them possibly being seen in the hippocampi, cerebellar or cerebral cortex, frontobasal and insular regions, basal ganglia, brainstem and, infrequently, the spinal cord. Those authors found that $50 \%$ of patients had normal brain MRI findings, and this is consistent with our results. As for EEGs, a study reported on in $2018^{19}$ found that the sensitivity of an abnormal EEG was high and that the first EEG recording was predictive of the final clinical outcome. The small amount of available data prevents us from discussing the differences in EEGs between the two groups in detail.

In a study of CSF findings in anti-NMDAR encephalitis, Wang et al. ${ }^{20}$ found that $39.5 \%$ of their patients exhibited increased CSF pressures, $58.1 \%$ had CSF pleocytosis, and 18.6\% had elevated CSF protein, but there was no relationship between changes in CSF findings and clinical outcomes. Our results are consistent with these findings, although we found that increased CSF pressure was related to poor responses to first-line treatments. However, Balu et al. ${ }^{6}$ found that both elevated CSF WBC count and CSF protein were associated with the 1-year outcome of AE. We found that 8 of our 29 patients were negative for NMDAR antibody in serum and none of them were negative in CSF, which verified the view of Wang et al. ${ }^{20}$ that detecting NMDAR antibodies is more sensitive in CSF than in serum. A previous study ${ }^{21}$ found that CSF and serum titers were higher in anti-NMDAR-encephalitis patients with poor outcomes than in those with good outcomes, whereas we found no differences between the remission and nonremission groups.

As a marker of systemic inflammation, the NLR is more reliable and stable than the WBC count for reflecting inflammatory responses. The NLR might be a useful marker for assessing the disease activity in neuromyelitis optica spectrum disorder, multiple sclerosis (MS), and systemic lupus erythematosus patients. ${ }^{22-24}$ Our team has recently reported that the NLR is a practical and reliable biomarker for monitoring disease progression in patients with $\mathrm{AE} .{ }^{9} \mathrm{In}$ the present study, the NLR was higher in the nonremission group before and after treatment, which may be attributed to the severe inflammatory response and the high probability of pulmonary infection in severe patients. Among the indicators of liver function and blood biochemistry, only AST was elevated in the nonremission group. We cannot conclude that patients with abnormal liver function have a reduced sensitivity to first-line treatments, since no significant difference in other liver function indicators. This might be attributable to drugs such as methylprednisolone being metabolized mainly in the liver. Previous studies ${ }^{25,26}$ have shown that the serum levels of UA and CysC were reduced in the acute phase of patients with anti-NMDAR encephalitis and were associated with the disease severity. However, we did not find differences in these parameters between the two groups in our study. We also found that the fibrinogen level was high in the nonremission group and that the patient who died presented with severe coagulopathy that eventually developed into diffuse intravascular coagulation. Fibrinogen is reportedly elevated in patients with MS and neuromyelitis optica. ${ }^{27}$ but the relationship between fibrinogen and anti-NMDAR encephalitis is unclear.

Six (54.55\%) of the patients in the nonremission group received second-line immunotherapy, which was consistent with the cohort study of Titulaer et al. ${ }^{5}$ Only one patient died, which was due to multiple- organ failure. The mortality rate was lower than that reported in the literature (5-7\%). 
This study was subject to some limitations, including the small number of patients and missing data such as for longterm EEG monitoring and laboratory indicators after the treatments. The retrospective design meant that some clinical data were incomplete. Prospective studies with larger cohorts are therefore needed to validate the findings reported here.

In conclusion, this study found that anti-NMDAR-encephalitis patients with more symptoms-especially involuntary movements, disturbance of consciousness, central hypoventilation, and accompanying hypoalbuminemia and pulmonary infection-may exhibit poor responses to first-line treatments. Positive second-line immunotherapy needs to be considered. Admission to an ICU, increased CSF pressure, and increased NLR are possible factors affecting the response to first-line treatments.

\section{Conflicts of Interest}

The authors have no potential conflicts of interest to disclose.

\section{Acknowledgements}

This study was supported by the Natural Science Foundation of China (Grant no. NSFC81671183).

\section{REFERENCES}

1. Dalmau J, Tüzün E, Wu HY, Masjuan J, Rossi JE, Voloschin A, et al. Paraneoplastic anti-N-methyl-D-aspartate receptor encephalitis associated with ovarian teratoma. Ann Neurol 2007;61:25-36.

2. Dalmau J, Lancaster E, Martinez-Hernandez E, Rosenfeld MR, BaliceGordon R. Clinical experience and laboratory investigations in patients with anti-NMDAR encephalitis. Lancet Neurol 2011;10:63-74.

3. Moscato EH, Peng X, Jain A, Parsons TD, Dalmau J, Balice-Gordon RJ. Acute mechanisms underlying antibody effects in anti-N-methylD-aspartate receptor encephalitis. Ann Neurol 2014;76:108-119.

4. Shin YW, Lee ST, Park KI, Jung KH, Jung KY, Lee SK, et al. Treatment strategies for autoimmune encephalitis. Ther Adv Neurol Disorder 2017;11:1756285617722347.

5. Titulaer MJ, McCracken L, Gabilondo I, Armangué T, Glaser C, Iizuka $\mathrm{T}$, et al. Treatment and prognostic factors for long-term outcome in patients with anti-NMDA receptor encephalitis: an observational cohort study. Lancet Neurol 2013;12:157-165.

6. Balu R, McCracken L, Lancaster E, Graus F, Dalmau J, Titulaer MJ. A score that predicts 1-year functional status in patients with anti-NMDA receptor encephalitis. Neurology 2019;92:e244-e252.

7. Broadley J, Seneviratne U, Beech P, Buzzard K, Butzkueven H, O'Brien T, et al. Prognosticating autoimmune encephalitis: a systematic review. J Autoimmun 2019;96:24-34.

8. Jang Y, Lee ST, Kim TJ, Jun JS, Moon J, Jung KH, et al. High albumin level is a predictor of favorable response to immunotherapy in autoimmune encephalitis. Sci Rep 2018;8:1012.

9. Zeng Z, Wang C, Wang B, Wang N, Yang Y, Guo S, et al. Prediction of neutrophil-to-lymphocyte ratio in the diagnosis and progression of autoimmune encephalitis. Neurosci Lett 2019;694:129-135.

10. Graus F, Titulaer MJ, Balu R, Benseler S, Bien CG, Cellucci T, et al. A clinical approach to diagnosis of autoimmune encephalitis. Lancet Neurol 2016;15:391-404.

11. Wang Y, Zhang W, Yin J, Lu Q, Yin F, He F, et al. Anti-N-methyl-d-aspartate receptor encephalitis in children of Central South China: clinical features, treatment, influencing factors, and outcomes. J Neuroimmunol 2017;312:59-65.

12. Zhang Y, Liu G, Jiang M, Chen W, He Y, Su Y. Clinical characteristics and prognosis of severe anti-N-methyl-D-aspartate receptor encephalitis patients. Neurocrit Care 2018;29:264-272.

13. Duan BC, Weng WC, Lin KL, Wong LC, Li ST, Hsu MH, et al. Variations of movement disorders in anti-N-methyl-D-aspartate receptor encephalitis: a nationwide study in Taiwan. Medicine (Baltimore) 2016; 95:e4365.

14. Vural A, Arsava EM, Dericioglu N, Topcuoglu MA. Central neurogenic hyperventilation in anti-NMDA receptor encephalitis. Intern Med 2012;51:2789-2792.

15. Waters KA, Machaalani R. Role of NMDA receptors in development of respiratory control. Respir Physiol Neurobiol 2005;149:123-130.

16. Fokkink WR, Walgaard C, Kuitwaard K, Tio-Gillen AP, Van Doorn $\mathrm{PA}$, Jacobs BC. Association of albumin levels with outcome in intravenous immunoglobulin-treated Guillain-Barré syndrome. JAMA Neurol 2017;74:189-196.

17. Chi X, Wang W, Huang C, Wu M, Zhang L, Li J, et al. Risk factors for mortality in patients with anti-NMDA receptor encephalitis. Acta Neurol Scand 2017;136:298-304.

18. Dalmau J, Tüzün E, Wu HY, Masjuan J, Rossi JE, Voloschin A, et al. Paraneoplastic anti-N-methyl-D-aspartate receptor encephalitis associated with ovarian teratoma. Ann Neurol 2007;61:25-36.

19. Sonderen AV, Arends S, Tavy DLJ, Bastiaansen AEM, Bruijn MAAM, Schreurs MWJ, et al. Predictive value of electroencephalography in anti-NMDA receptor encephalitis. J Neurol Neurosurg Psychiatry 2018; 89:1101-1106.

20. Wang R, Guan HZ, Ren HT, Wang W, Hong Z, Zhou D. CSF findings in patients with anti-N-methyl-D-aspartate receptor-encephalitis. Seizure 2015;29:137-142.

21. Gresa-Arribas N, Titulaer MJ, Torrents A, Aguilar E, McCracken L, Leypoldt $\mathrm{F}$, et al. Antibody titres at diagnosis and during follow-up of anti-NMDA receptor encephalitis: a retrospective study. Lancet Neurol 2014;13:167-177.

22. Lin J, Xue B, Li J, Xu H, Huang X, Yao Z, et al. Neutrophil to lymphocyte ratio may be a helpful marker to evaluate disease activity in NMOSD. Neurol Sci 2017;38:1859-1863.

23. Wu Y, Chen Y, Yang X, Chen L, Yang Y. Neutrophil-to-lymphocyte ratio (NLR) and platelet-to-lymphocyte ratio (PLR) were associated with disease activity in patients with systemic lupus erythematosus. Int Immunopharmacol 2016;36:94-99.

24. Bisgaard AK, Pihl-Jensen G, Frederiksen JL. The neutrophil-to-lymphocyte ratio as disease actvity marker in multiple sclerosis and optic neuritis. Mult Scler Relat Disord 2017;18:213-217.

25. Shu Y, Wang Y, Lu T, Li R, Sun X, Li J, et al. Serum uric acid and antiN-methyl-D-aspartate receptor encephalitis. Neurochem Int 2017;108: 34-39.

26. Shu Y, Chang Y, Wu H, Li J, Cao B, Sun X, et al. Serum cystatin C and anti-N-methyl-D-aspartate receptor encephalitis. Acta Neurol Scand 2018;137:515-522.

27. Zhang Y, Zhang X, Liu D, Wang H, Pan S, Wang D, et al. Elevated fibrinogen levels in neuromyelitis optica is associated with severity of disease. Neurol Sci 2016;37:1823-1829. 